\title{
Using gamification to incentivize sustainable urban mobility.
}

\author{
KAZHAMIAKIN, R., MARCONI, A., PERILLO, M., PISTORE, M., VALETTO, \\ G., PIRAS, L., AVESANI, F. and PERRI, N.
}

(C) 2015 IEEE. Personal use of this material is permitted. Permission from IEEE must be obtained for all other uses, in any current or future media, including reprinting/republishing this material for advertising or promotional purposes, creating new collective works, for resale or redistribution to servers or lists, or reuse of any copyrighted component of this work in other works. 


\section{Using Gamification to Incentivize Sustainable Urban Mobility}

\author{
Raman Kazhamiakin, Annapaola Marconi, \\ Mirko Perillo, Marco Pistore, Giuseppe Valetto \\ Fondazione Bruno Kessler \\ Trento, Italy 38123 \\ Email: \{raman, marconi, \\ mirko.perillo, pistore, valetto\}@fbk.eu
}

\author{
Luca Piras \\ Trento RISE \\ Trento, Italy 38123 \\ Email: luca.piras@trentorise.eu
}

\begin{abstract}
Sustainable urban mobility is an important dimension in a Smart City and one of the key issues in National and International Programmes. However, innovative and often costly mobility policies and solutions introduced by cities are liable to fail, if not combined with initiatives aimed at increasing the awareness of citizens, and promoting their behavioural change. This paper explores the potential of gamification mechanisms to incentivize voluntary behavioural changes towards sustainable mobility solutions. We present a service-based gamification framework, developed within the STREETLIFE EU Project, which can be used to develop games on top of existing services and systems within a Smart City, and discuss the empirical findings of an experiment conducted in the city of Rovereto on the effectiveness of gamification to promote sustainable urban mobility.
\end{abstract}

\section{INTRODUCTION}

Facilitating and promoting more sustainable mobility means and habits is an objective of increasing importance for cities across the globe. Sustainable urban mobility has captured a lot of attention as one among the principal dimensions in a Smart City [1]. Leveraging advanced ICT assets to help reaching that objective is a significant challenge and, at the same time, a great opportunity to make a city, its citizens and its governance institutions smarter, as advocated in [2].

The promotion of Voluntary Travel Behavior Change (or VTBC) has been indicated as a key issue for sustainable urban mobility in the scientific literature [3], [4], as well as in official national and international policy documents [5]; it is also tightly coupled with the definition and execution of city-wide mobility policies. On the one hand, policies that target sustainability - and the corresponding smart mobility services - can be successful only if they are embraced by citizens in a convinced and continued way; on the other hand, city policies that create and manage effective incentives can help to break citizens' habits and affect their mobility choices, boost acceptance, and, ultimately, make a difference in the urban environment.

We have been developing a Smart City service platform, which operates on both sides of that equation ${ }^{1}$. We provide mechanisms to embed and implement city-level policies within a set of smart mobility services provisioned by our platform, such as journey planning and route recommendation services

\footnotetext{
${ }^{1}$ In the context of the STREETLIFE European project - http://streetlifeproject.eu
}

that are routinely used by citizens. Moreover, we provide mechanisms to incentivize citizens to take choices in accord with those mobility policies, by means of gamification. We have developed a generic, service-based gamification framework, which enables to design, deploy and execute games that predicate on how citizens use the repertoire of Smart City services provisioned by - or simply interfacing with - our platform. This enables a gamification designer to conceive games that augment the citizens awareness of existing and new sustainable mobility polices and services in the city, and motivates them to embrace the corresponding enabling ICT solutions, in order to gain status and reputation in the game, and earn rewards (either virtual or material).

In this paper, we describe the results of our approach, by illustrating a case study we have conducted in the Fall of 2014 in the city of Rovereto, Italy, describing the gamification affordances we have developed for that case study, and reporting the empirical findings related to the sustainable urban mobility outcomes. The results show the potential of gamification to support VTBC and indicate how a gamification framework like the one we are developing can become an important asset for the promotion of sustainable behaviors and city-wide policies related to the services made available within a complex sociotechnical system like a Smart City.

The rest of the paper is structured as follows: in Section II we introduce the main concepts of gamification, report on known uses in the domain of sustainable urban mobility, and indicate some recent works on frameworks and technologies to support the development of gamification solutions; in Section III we provide a technical overview of our service-based gamification framework; in Section IV we outline the context and content of our case study in Rovereto, and discuss the experiments we have carried out; in Section V we discuss the most interesting empirical results that we have extracted from the case study. Finally, in Section VI we offer some reflections on the lessons we have learned and on future work directions on the topic of gamification in Smart Cities.

\section{RELATED WORK}

Gamification is often defined as "the use of game design elements in non-game contexts" [6]. Nowadays, gamification is leveraged to stimulate specific usage patterns by users or customers of an ICT system in some business domain, by 
injecting mechanisms and concepts typical of games within the system, even if it was not originally designed with playful intentions in mind [7]. Among the most commonly used gamification elements there are points, badges and leaderboards; more advanced ones include levels, paths, challenges, stories, feedback, and user powers [6], [8].

Gamification is a methodology that has been used successfully in many domains [9]. It leverages fun, competition, rewards and game mechanics in ICT-mediated contexts for marketing, or to induce workers to carry out particular tasks and reach specific objectives within an enterprise, or to incentivize virtuous behavioral changes in society at large (for example, it can help to motivate people to have regular physical activity [10], or to achieve savings in energy consumption [11]).

Gamification is relevant for Smart Cities, as it can be leveraged to affect citizens' behaviour in relation to many major smart city concerns, such as participatory governance, tourism, culture, education, mobility, etc. Our work on promoting citydefined sustainable mobility polices and citizens' VTBC is in the area of gamification for smart and sustainable urban mobility. Some other recent works have reported promising results in this area. Merugu et al. [12] illustrated an application to reduce traffic congestion, while Hoh et al. [13] coupled gamification and crowdsourcing, in order to involve citizens in sharing information about parking spaces in the city. Gabrielli et al. describe design methodologies and lessons learned, based upon gamification case studies carried out in the cities of Trento (Italy), Milan (Italy), Helsinki (Finland) and Barcelona (Spain) [14], [15]. Buningh et al. [16] successfully implemented in Eindhoven (The Netherlands), a gamified system for stimulating company employees to choose sustainable means of commuting to work.

While gamification offers considerable promise to sustainable urban mobility and Smart Cities in general, its implementation for a given context remains difficult and expensive. Most applications are tightly coupled to the specific business logic they wish to gamify, which hinders both evolution and reuse. Furthermore, they often require the gamification expert to redefine many of the concepts of the business domain in a gamified form, which leads to information duplication. For these reasons, in the last few years research has begun to focus on gamification frameworks, that is, platforms and tool sets that can help making the design and development of gamification applications easier, faster and cheaper.

Notable works in this area include Herzig et al. [17], which discusses a platform to gamify Enterprise Information Systems, and a specification and modeling language - called GaML [18], [19] - to facilitate the definition of games. Monterrat et al. [20] present a gamification framework for the e-learning domain, which also strives to decouple gamification concepts from the underlying system. Their approach, however, impacts primarily the presentation of game elements to the user of the e-learning system, that is, the separation occurs only at the level of the user interface of the gamified application.

In our work, we aim at building a generic gamification framework that accomplishes both generality and extensibility, by leveraging the service-oriented paradigm. The key aspect of our framework is to be able to integrate in a wide array of games the extremely large (and ever-growing) number and variety of ICT systems, applications and services that exist in a smart city. Our ultimate goal is to provide tools to gamify a smart city [21], and facilitate the deployment of games which incentivize players to make use of those diverse ICT resources in ways that are beneficial to the community, thus becoming active participants in the governance of the smart city.

\section{TECHNICAL SOLUTION}

We are developing a gamification framework, which must support the specification and execution of a wide variety of games within a Smart City. Figure 1 shows the main building blocks of our framework, i.e., a Gamification Engine (or GE) for the instantiation and execution of games; a wrapping layer that manages the interactions between the GE and any involved ICT systems of the Smart City; a game presentation layer that indicates to players their game progression; an extensible set of gamification plugins, which represent typical concepts that can be used in defining a game, and which encapsulate logic and idiosyncratic properties specific to each concept.

A Smart City is an eminently open-boundary system, in which new and heterogeneous ICT affordances - ranging from new information systems, to sensors, to cyber-physical systems, to services, to terminals, to apps, etc. - can become available at any given time. A framework that wants to cope with (and take advantage of) this variety and dynamism must have the characteristics of openness and generality. For this reason, we have built our gamification framework upon the unifying abstraction of service, and in accord with the architectural principles of service orientation. Service concepts and technologies are prevalent and ubiquitous in a Smart City; we assume that the resources, systems and applications that must be involved in each of the games that are developed with our framework expose game-relevant features as services (or, at least, in a way that makes possible to wrap them as services).

This is the purpose of the wrapping layer in Figure 1. Wrapping consists in integrating a lightweight Listener service, which issues notifications whenever game-relevant actions, which we call gamifiable actions are taken by the citizen/player, or otherwise occur on the wrapped systems. It is the responsibility of the gamification designer to designate such gamifiable actions and the content of the corresponding notifications. The general form of a notification is

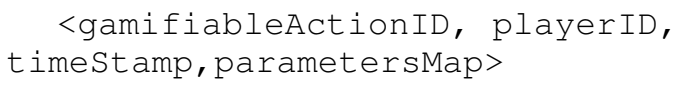

where gamifiableActionID is a unique ID and the parametersmap contains a set of key/value pairs that are specific to that gamifiable action. The wrapping layer issues notifications on behalf of the wrapped ICT systems through a simple actionPerformed service interface exposed by the GE. Moreover, the wrapping layer enables strongly decoupled interactions between the native Smart City functionalities involved in a specific game and the GE, which is the component responsible to execute that game and managing its status.

The core of the GE is a rule execution system, in which one or more games may run concurrently. In our current prototype we use the Open Source DROOLS ${ }^{2}$ rule engine. Each game is thus represented by a rule set, which implements the desired

\footnotetext{
${ }^{2}$ http://www.drools.org
} 


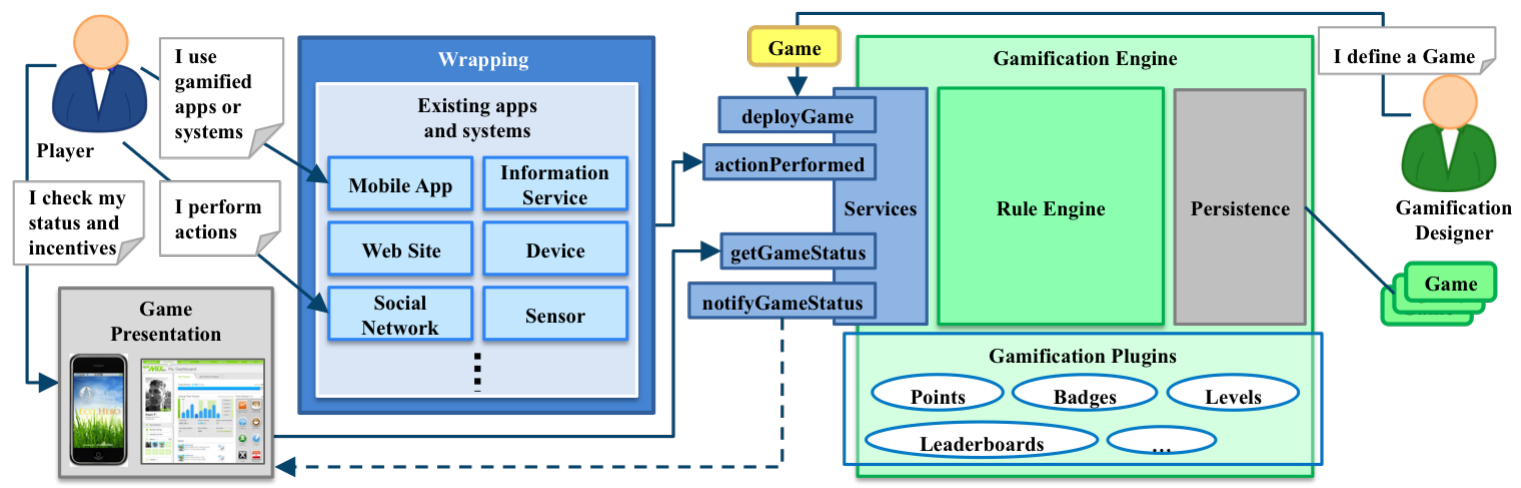

Fig. 1. Architecture of the gamification framework.

game logic, which has been specified in advance by the gamification designer, and deployed onto the rule engine runtime. Game rules are fired in response to the gamifiable actions received through the service interface actionPerformed. Gamifiable actions serve as triggers for the game logic rules, which predicate upon an in-memory object base. This object base represents the game state for each participating player, as well as any global state the game may need to maintain, and is populated by instantiating game plugins. A game plugin is a service-oriented module that represent a game concept and manages all instances of that game concept; it exposes service operations that can be used to interact with the game concept, and implements them in compliance with the concept's game semantics. As a very simple example, the plugin for the Badge Collection concept exposes operations to assign (and possibiy revoke) a badge to a player and insert it in the collection; also, since it does not make sense for a player to collect the same badge repeatedly, the implementation of the assign operation of the BadgeCollectionPlugin includes a guard to check whether the player has already earned that badge.

The game state evolves as the right-hand side of the game rules manipulates the object base through the plugins services. Besides changing the state of the game, rules can also issue additional notifications; these notifications can be consumed by the GE rule system. To continue the example above, the completion of a badge collection can cause a new event, which is consumed by a Player Level concept, to move the player to the next game level. That in turn, can cause an event that unlocks new game elements such as a new Power or Challenge.

Events generated internally to the GE can also be issued to external wrapped systems, including user Apps (e.g., for pushing notifications of game achievements to the players). That is one mechanism that supports the presentation of the game state to players. The presentation layer of the framework also offers a pull mechanism, which queries the GE through a getGameStatus service, and retrieves information specific to the various plugins. This can be used to generate dynamic Web pages that offer a view of the game state (for example points, leaderboards, or player's achievements, etc.). Furthermore, the Game Presentation layer can similarly be used to issue notifications (not ifyGamestatus service) on the part of the GE; this is especially useful to communicate with players via interactive modes, for example to issue alerts, invitations to challenges, or other game-relevant events.
The final responsibility of the gamification engine is the game persistence. That entails saving the rule base as well as checkpointing, separately, the game instances in execution, i.e. all players' states plus any global state of each game. Our persistence layer is implemented as a NoSQL database.

\section{CASE STUDY}

Rovereto is a medium-small city of about 40.000 inhabitants situated in the North-East of Italy. Despite its small size in comparison to other European municipalities, the city is exposed to a significant traffic pressure especially in the city centre. On average, each two inhabitants, one owns a car, and if we consider the modal split, the percentage of trips travelled by private vehicles is 59\% [22]. Moreover, parked cars occupy high valued space in the city centre that could be used by pedestrians and cyclists. The occupancy rate of parking areas in certain central zones reaches $90 \%$, and this generates additional traffic related to cars travelling and looking for a parking space.

The city invested considerable effort and resources in improving the mobility situation. For instance, Rovereto has more than $30 \mathrm{~km}$. of bicycle lanes and in the future the total length of bicycle network is foreseen to be almost $75 \mathrm{~km}$. In addition, in September 2014, Rovereto launched a new bike sharing system with more than 10 stations.

In Fall 2014, within the STREETLIFE EU Project, we conducted an experiment to evaluate the impact of sustainable mobility recommendations and gamification incentives on the mobility behavior of commuters that need to travel routinely to the center of the city by car. For the experiment, we recruited a group of 40 users, who responded to invitations we sent to all people who pay a yearly fee for on-street parking permits in the central area of Rovereto, but reside outside the center.

At the beginning of the experiment, we met the participants and instructed them to use our routing mobile App Viaggia Roveretgoto $^{3}$ for their daily itinerary planning, so that we could $\log$ and store their mobility behavior during the experiment. The experiment spanned 5 weeks, and consisted of three phases. In phase 1 - baseline (1 week) the end users had to simply familiarize with the App and record their regular

\footnotetext{
${ }^{3}$ Available on Google Play at https://play.google.com/store/apps/details?id= eu.trentorise.smartcampus.viaggiarovereto\&hl=en.
} 
itineraries and normal mobility behavior. In phase 2 - recommendations without gamification (2 weeks) we switched on an additional Viaggia Rovereto functionality, i.e., sustainable mobility recommendations. The App started to rate the alternative routes for each itinerary requested, based on sustainability policies defined in accord with the mobility management of the city of Rovereto, and configured within our journey planner service; the App would then present the highest-rated options by highlighting them in green to the user and placing them in the first positions at the top of the list (see Fig.2). The policies we implemented aimed at pushing those itinerary solutions that - besides having a low carbon impact - would either include Park\&Ride solutions or promote the new bike-sharing service.

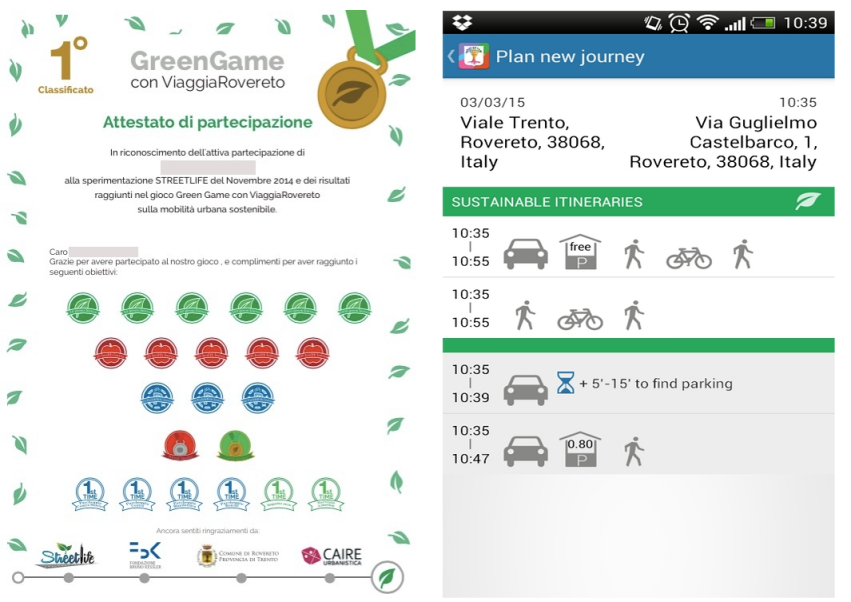

Fig. 2. Certificate of attendance with game results (left) and recommendations from Viaggia Rovereto App (right).

In phase 3 - gamification (2 weeks) we introduced gamification on top of the features of phase 2, and we launched Green Game with ViaggiaRovereto. The game included three separate types of points (with the corresponding leaderboards): Green Points (related to $\mathrm{Km}$. travelled with sustainable transportation means), Health Points (related to $\mathrm{Km}$. travelled biking or walking) and Park\&Ride points (related to repeated usage of Park\&Ride facilities). We also implemented a variety of badges and badge collections, some of which were linked to reaching certain amounts of points in the three categories above, or occupying a prominent position in one of the leaderboards, while others (including some "surprise badges") were awarded when the user took specific kind of trips, or explored some mobility alternatives (e.g. when they used the designated Park\&Ride facilities for the first time, tried the Bike Sharing service for the first time, etc.).

At the end of the game, all participants received a certificate of attendance, reporting personal game achievements, in terms of gained badges and points (see Fig.2). The three best players were rewarded with a one-month free pass to the new bike sharing service.

Across the three phases of the experiment, a total of 36 people made use of the system for planning their urban mobility itineraries. In phase 1 there were 26 active participants; in phase 2 there were 29; and in phase 3 there were 26 players in Green Game with ViaggaRovereto. There were 20 participants who were active in all of the three experiment phases.

\section{Evaluation}

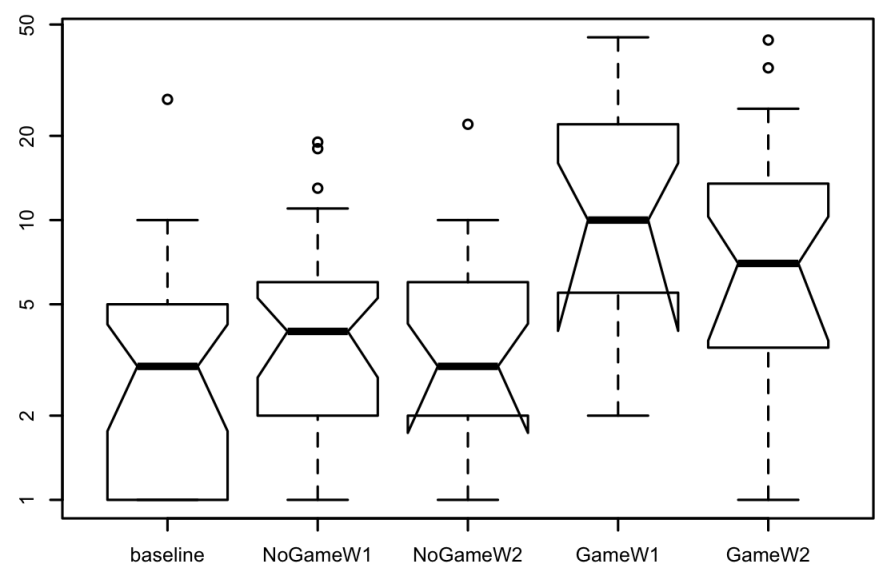

Fig. 3. Distribution of saved trip per user during the experiment.

The case study described in Section IV has allowed us to collect a wealth of data on mobility behavior of participants and Rovereto citizens. We report here some of the most significant results that have to do with the effect of gamification, that is, how the mobility behavior of players taking part in the game in phase 3 of the experiment changed with respect to the other two phases. Hereby we present a selection of our evaluation results, which is limited for space reasons, and which addresses the following research questions:

- RQ1: did the introduction of gamification encourage players to use the ViaggiaRovereto mobile App more frequently for urban mobility planning needs?

- RQ2: did the introduction of gamification incentivize players to select prevalently those route alternatives that were recommended by our system?

- RQ3: if RQ2 is verified, how much did that form of VTBC impact the mode choices of the players towards more sustainable transport modes?

To address RQ1, we compared the distribution of the number of trips per user that were selected and saved by participants, in each of the five weeks of the experiment, as an indicator of how often users consult and make use of the App and the journey planning service; the hypothesis is that during the game weeks the App was consulted more frequently. The boxplot chart in Figure 3 assesses the changes in the distribution over those five weeks. The increment in App usage during the game weeks compared to the baseline and non-game weeks is visually evident. To better understand the level of statistical significance of that difference, and since the distributions could not be considered normal, we have carried out non-parametric Mann-Withney tests among the 5 weeks; for lack of space we cannot report the full crosstabs; however, the results always indicate that the distributions of game weeks (4 and 5) are significantly different (greather than) the distributions of the other weeks, whereas the disitributions of Weeks 1 to 3 are not statistically different.

Those results are consistent with the hypothesis that GreenGame with ViaggiaRovereto has incentivized its players to make use of the ViaggiaRovereto App more frequently, and hence support a positive answer to RQ1. 
To address RQ2, we compared the proportion of the routes chosen by users that were recommended by the ViaggiaRovereto App as sustainable during phase 3 vs. phase 2 (notice that a comparison with phase 1 was not available, because this feature was not active during the baseline phase); the hypothesis is that the game encouraged players to follow the sustainable mobility recommendations offered by the App. As shown in Table I, during phase 2, the experiment participants selected sustainable routes for $42.7 \%$ of their itinerary choices. In phase 3 , they selected sustainable routes $60.6 \%$ of the times.

TABLE I. SUSTAINABLE MOBILITY RECOMMENDATION ACCEPTANCE.

\begin{tabular}{|l|c|c|}
\hline & phase 2 (no game) & phase 3 (game) \\
\hline sustainable trips & 105 & 323 \\
\hline total trips & 246 & 533 \\
\hline \hline ratio & 42.7 & 60.6 \\
\hline
\end{tabular}

We carried out a statistical proportion test on this data, to see whether the higher proportion in phase 3 is statistically significant. The results of that test are:

$$
\mathrm{X} \text {-square }=21.1096 \mathrm{p} \text {-value }=2.169 \mathrm{e}-06
$$

The higher proportion of sustainable route choices in phase 3 is therefore very significant statistically, which supports a positive answer to RQ2. This result is in line with our principal work hypothesis, i.e., gamification in a Smart City can be effective to instigate VTBC in the direction of more sustainable urban mobility.

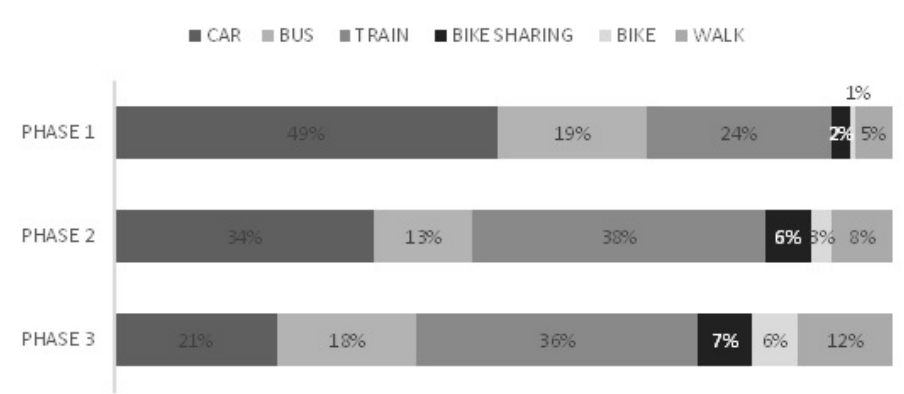

Fig. 4. Percentage of $\mathrm{Km}$ travelled for each mode of transport.

To address RQ3, we tried to assess whether the VTBC we observed had in fact a positive effect, i.e., promoted the more sustainable modes of urban transportation in Rovereto. For that, we looked at changes in mode choice by the participants across the three phases. The hypothesis here is twofold: a) the activation of sustainable mobility recommendations in phase 2 caused significant mode shift towards sustainability, with respect to the typical mobility habits captured in phase 1; and b) the activation of gamification in Phase 3 further improved those mode shifts with respect to phase 2 .

Figure 4 reports the relative share of the total $\mathrm{Km}$. travelled for the six modes available to Rovereto commuters (individual car, public transport buses, train, the new bike sharing service, private bycicle, and walking), and visually shows the shifts that occurred among experiment phases. In the rest of this section, we analyze in depth the significance of the shift for the car mode and the bike sharing mode, since those two modes were explicit targets of the city mobility management policies, which we strived to support. To analyze such hypothesized mode shifts, we looked first of all at the proportion of itineraries involving these two modes vs. the total number of itineraries recorded in each phase.

TABLE II. TRIPS USING BIKE SHARING AND CAR MODES.

\begin{tabular}{|l|c||c|c|}
\hline & phase 1 (baseline) & phase 2 (no game) & phase 3 (game) \\
\hline total trips & $\mathbf{1 1 5}$ & $\mathbf{2 4 6}$ & $\mathbf{5 3 3}$ \\
\hline car trips & $40(34.8 \%)$ & $67(27.2 \%)$ & $90(16.9 \%)^{* * * *}$ \\
\hline bike sharing trips & $13(11.3 \%)$ & $51(20.7 \%)^{*}$ & $115(21.6 \%)$ \\
\hline
\end{tabular}

Table II recaps the percentage of chosen itineraries that involved at least one leg in either the car or bike sharing mode. We carried out proportion tests to understand the significance of the mode shift between each phase and the previous one. The findings obtained are the following:

- although there was a shift towards less frequent car usage between phase 1 and phase 2, that was not pronounced enough to be statistically significant given the sample (the p-value was 0.09); however, the further shift towards less car usage during the game phase was very statistically significant, even with respect to the already reduced car usage observed in phase 2 ;

- $\quad$ there was a moderately significant shift towards more bike sharing usage between phases 1 and 2 ; that can be interpreted as users willing to accept the App recommendations to leverage the new bike sharing service for their mobility needs. That trend remained practically the same in phase 3 ; therefore, it looks like our game did not provide sufficient additional incentives to use bike sharing.

Taken together, those results point in the direction that gamification incentivized players to use their private cars less, which supports a positive answer to RQ3. To further validate these findings, we then looked at the percentage of $\mathrm{Km}$. travelled by each experiment participant in the bike sharing mode and car mode during each phase, and evaluated the difference between the corresponding distributions. Since those distributions are not normal, we used a non-parametric pairwise Wilcoxon text; the hypothesis is that the introduction of gamification induced a significant shift in the distributions (greater for bike sharing usage and lesser for car usage, respectively). For both the bike sharing and the car mode, the considered population was the subset of participants who used that particular mode in either phase 2 or phase 3; this allowed us to eliminate participants that did never make use of (or did not have available) that mode of transportation.

For the bike sharing mode, we tested the distribution difference between phase 2 and phase 3 :

$$
(\mathrm{n}=16) \quad \mathrm{V}=80 \quad \mathrm{p} \text {-value }=0.2809
$$

The high $\mathrm{p}-$ value indicates that any shift in the percentage of $\mathrm{Km}$. travelled by bike sharing is not statistically significant. For comparison, we also investigated the same difference in distribution for theose experiment participants who used bike sharing in both phase 1 vs. phase 2 :

$$
(\mathrm{n}=15) \quad \mathrm{V}=10 \quad \mathrm{p} \text {-value }=0.02272
$$

The result above denotes a modest but moderately significant shift, which reinforces our previous observation that the sustainable mobility recommendations in phase 2 improved the 
usage of bike sharing, while the game introduced in phase 3 was not able to further augment the situation in that respect.

For the car mode, when we tested the distribution difference between phases 2 and 3, we obtained the following result:

$$
(\mathrm{n}=13) \quad \mathrm{V}=13 \quad \mathrm{p} \text {-value }=0.01074
$$

which denotes a statistically significant shift towards less car traveling during the game. Since the analogous test between phase 1 and phase 2 did not produce significant results, it can be said that the introduction of gamification incentivized users to travel less with their private car, and hence supports a positive answer to RQ3.

Finally, a word on the private cycling and walk modes, which are both visibly boosted in Figure 4. We do not fully report on them here, since they were not the principal concerns in our game; however, while the shift in the walk mode is not sufficiently pronounced across phases to be statistically significant, the shift in private cycling can be seen as a positive - although unplanned - by-product of game participation, since it shows a statistically significant difference only between phases 2 and 3 .

\section{CONCLUSION}

Our case study demonstrated the potential of gamification to promote sustainable urban mobility policies and VTBC. In particular, the introduction of a game in a controlled experiment in Rovereto (Italy) has increased the reliance of the game participants on the ICT mobility services we have deployed in the city, their willingness to accept and try the recommendations provided by those service to use new and more sustainable transportation options for their daily commuting needs, and caused a significant shift towards those options, based on the percentage of $\mathrm{Km}$. travelled by participants in the various transport modes.

In the next future, we will continue to work on our generic service-based framework for the design and development of gamification applications for Smart Cities. We will evaluate our progress in the context of further smart urban mobility experiments; we have planned an open-field, large-scale, long-running test in Rovereto, and an experiment based on a green mobility competition among local private companies.

\section{ACKNOWLEDGMENT}

This work was supported in part by the European Commission under Grant Agreement \# 608991. We would like to thank our partners in the STREETLIFE consortium. Special thanks to the City of Rovereto and its administration for their invaluable help in this work.

\section{REFERENCES}

[1] R. Giffinger, G. Haindlmaier, and H. Kramar, "The role of rankings in growing city competition," Urban Research \& Practice, vol. 3, no. 3, pp. 299-312, 2010.

[2] T. Nam and T. Pardo, "Conceptualizing smart city with dimensions of technology, people, and institutions," in Proc. of the 12th Annual International Digital Government Research Conference: Digital Government Innovation in Challenging Times. ACM, 2011, pp. 282-291.
[3] W. Brög, E. Erl, I. Ker, J. Ryle, and R. Wall, "Evaluation of voluntary travel behaviour change: Experiences from three continents," Transport Policy, vol. 16, no. 6, pp. 281-292, 2009.

[4] D. Banister, "The sustainable mobility paradigm," Transport Policy, vol. 15 , no. 2 , pp. $73-80,2008$.

[5] European Commission, "Roadmap to a single european transport area - towards a competitive and resource efficient transport system," 2011.

[6] S. Deterding, D. Dixon, R. Khaled, and L. Nacke, "From game design elements to gamefulness: Defining "gamification"," in Proc. of the 15th International Academic MindTrek Conference: Envisioning Future Media Environments, ser. MindTrek '11. ACM, 2011, pp. 9-15.

[7] S. Deterding, M. Sicart, L. Nacke, K. O'Hara, and D. Dixon, "Gamification. using game-design elements in non-gaming contexts," in Proc. of the 2011 Conference on Human Factors in Computing Systems. ACM, 2011, pp. 2425-2428.

[8] G. Zichermann and C. Cunningham, Gamification by Design: Implementing Game Mechanics in Web and Mobile Apps. "O'Reilly Media, Inc.", 2011.

[9] J. Hamari, J. Koivisto, and H. Sarsa, "Does gamification work?a literature review of empirical studies on gamification," in System Sciences (HICSS), 2014 47th Hawaii International Conference on. IEEE, 2014, pp. 3025-3034.

[10] G. Walsh and J. Golbeck, "Stepcity: A preliminary investigation of a personal informatics-based social game on behavior change," in $\mathrm{CHI}$ '14 Extended Abstracts on Human Factors in Computing Systems, ser. CHI EA '14. ACM, 2014, pp. 2371-2376.

[11] B. Cowley, J. Moutinho, C. Bateman, and A. Oliveira, "Learning principles and interaction design for 'green my place': A massively multiplayer serious game," Entertainment Computing, pp. 103-113, 2011.

[12] D. Merugu, B. Prabhakar, and N. Rama, "An incentive mechanism for decongesting the roads: A pilot program in bangalore," in Proc. of ACM NetEcon Workshop, 2009.

[13] B. Hoh, T. Yan, D. Ganesan, K. Tracton, T. Iwuchukwu, and J. Lee, "Trucentive: A game-theoretic incentive platform for trustworthy mobile crowdsourcing parking services," in Intelligent Transportation Systems (ITSC), 2012 15th International IEEE Conference on, 2012.

[14] S. Gabrielli, R. Maimone, P. Forbes, J. Masthoff, S. Wells, L. Primerano, L. Haverinen, G. Bo, and M. Pompa, "Designing motivational features for sustainable urban mobility," in CHI'13 Extended Abstracts on Human Factors in Computing Systems. ACM, 2013, pp. 1461-1466.

[15] S. Gabrielli, P. Forbes, A. Jylhä, S. Wells, M. Sirén, S. Hemminki, P. Nurmi, R. Maimone, J. Masthoff, and G. Jacucci, "Design challenges in motivating change for sustainable urban mobility," Computers in Human Behavior, vol. 41, pp. 416-423, 2014.

[16] S. Buningh, R. Martijnse-Hartikka, and J. Christiaens, "Mobi-modal shift through gamification," in Transport Research Arena (TRA) 5th Conference: Transport Solutions from Research to Deployment, 2014.

[17] P. Herzig, M. Ameling, and A. Schill, "A generic platform for enterprise gamification," in Software Architecture (WICSA) and European Conference on Software Architecture (ECSA), 2012 Joint Working IEEE/IFIP Conference on. IEEE, 2012.

[18] P. Herzig, K. Jugel, C. Momm, M. Ameling, and A. Schill, "Gaml-a modeling language for gamification," in Proc. of the 2013 IEEE/ACM 6th International Conference on Utility and Cloud Computing. IEEE Computer Society, 2013.

[19] A. Matallaoui, P. Herzig, and R. Zarnekow, "Model-driven serious game development integration of the gamification modeling language gaml with unity," in System Sciences (HICSS), 2015 48th Hawaii International Conference on. IEEE, 2015, pp. 643-651.

[20] B. Monterrat, É. Lavoué, and S. George, "Motivation for learning: Adaptive gamification for web-based learning environments," in Proc. of the 6th International Conference on Computer Supported Education (CSEDU), 2014, pp. 117-125.

[21] M. Sakamoto, T. Nakajima, and S. Akioka, "A methodology for gamifying smart cities: Navigating human behavior and attitude," in Distributed, Ambient, and Pervasive Interactions. Springer, 2014, pp. 593-604.

[22] Comune di Rovereto, "Carta della circolazione dei cittadini: Mobilitiamoci," 2011. 